\title{
RECUPERAÇÃO DE COMPOSTOS DE IODO DE REAGENTES E SOLUÇÕES LABORATORIAIS
}

\author{
Vanessa da Matta dos Santos e Júlio Carlos Afonso* \\ Departamento de Química Analítica, Instituto de Química, Universidade Federal do Rio de Janeiro, Av. Athos da Silveira Ramos,
} 149, Bl. A, 21941-909 Rio de Janeiro - RJ, Brasil

Recebido em 18/3/11; aceito em 1/7/11; publicado na web em 16/8/11

\begin{abstract}
RECOVERY OF IODINE COMPOUNDS FROM LABORATORY CHEMICALS AND SOLUTIONS. This work presents simple routes to recover iodine compounds from oxidized laboratory chemicals and aqueous solutions (HI and $\mathrm{KI}$ ) used in laboratory chemistry classes. These routes are based on the oxidation of iodide ions $\left(\mathrm{I}^{-}\right)$to iodine $\left(\mathrm{I}_{2}\right)$ by an oxidant $\left(\mathrm{H}_{2} \mathrm{O}_{2}\right)$ or reduction of oxidized iodine by red phosphorus or hydrazine. Both routes presented high yields. The oxidative route is of general use whereas the reductive one is appropriate for restoring original iodine reagents. Final wastes were generated in low amounts. This work is appropriate for teaching many laboratory techniques (e.g., distillation, titration and filtration) in the chemical laboratory.
\end{abstract}

Keywords: iodine; waste treatment; element recovery.

\section{INTRODUÇÃO}

No início do século XIX, o químico francês Bernard Courtois (1777-1838) trabalhava na obtenção de sais de sódio e potássio a partir da queima de diversas algas abundantes ao largo do litoral da Normandia e da Grã-Bretanha, seguida de tratamento das cinzas com água. Por evaporação da solução os sais $\mathrm{NaCl}, \mathrm{KCl}$ e $\mathrm{K}_{2} \mathrm{SO}_{4}$ eram obtidos nesta ordem. A solução residual continha cloretos, brometos, iodetos, carbonatos, sulfatos e produtos de redução deste último (polissulfetos, sulfitos, tiossulfatos). Courtois tratava essa solução com ácido sulfúrico para eliminar esses produtos de redução. Em 1811, acidentalmente adicionou mais ácido sulfúrico do que deveria; para sua surpresa, surgiram vapores de coloração violeta e um odor irritante. ${ }^{1}$ Ao entrar em contato com superfícies frias, os vapores formavam cristais negros com um brilho metálico. Após alguns experimentos, Courtois notou que a nova substância se combinava com hidrogênio, fósforo e alguns metais, e não regia facilmente com carbono e oxigênio. A mistura da substância com amônia era explosiva. Ele então suspeitou que havia um novo elemento. ${ }^{1}$ Charles-Bernard Désormes (1771-1862) e Nicolas Clément (1779-1842) prosseguiram os trabalhos com essa nova substância. Clément cedeu amostras para LouisJoseph Gay-Lussac (1778-1850) e Humphry Davy (1778-1829). Em 1813 e 1814, os trabalhos publicados por Davy e Gay-Lussac indicavam que as propriedades do novo elemento eram semelhantes àquelas do cloro. ${ }^{1,2} \mathrm{~A}$ denominação iodo (do grego iodes, violeta) é uma alusão aos vapores do elemento.

$\mathrm{O}$ iodo é um elemento raro na crosta terrestre $\left(0,14 \mathrm{mg} \mathrm{kg}^{-1}\right) \mathrm{e}$ na água do mar $\left(0,05 \mathrm{mg} \mathrm{L}^{-1}\right)$, sendo muito menos abundante que os demais halogênios (exceto o astato). ${ }^{3}$ É obtido a partir de cinzas de algas marinhas (as quais contêm iodeto, $\mathrm{I}^{-}$) e das águas-mães do processamento do Salitre do Chile (que contêm iodato, $\mathrm{IO}_{3}^{-}$) e outras salmouras. ${ }^{2} \mathrm{O}$ íon I- é oxidado a iodo eletroliticamente ou sob aquecimento com dióxido de manganês em meio de ácido sulfúrico (reação 1). Os iodatos são reduzidos a iodo mediante tratamento com hidrogenossulfito de sódio $\left(\mathrm{NaHSO}_{3}\right.$, reação 2) ${ }^{2,4}$ Ele pode ser purificado por sublimação, obtendo-se iodo de elevadíssima pureza. O Brasil não produz iodo, importando-o principalmente do Chile para suprir suas necessidades internas. A produção mundial em 2010 ficou em torno de 29.000 t. Chile (60\%) e Japão (30\%) são

\footnotetext{
*e-mail: julio@iq.ufrj.br
}

os principais produtores, seguidos de China, Arzebaijão, Rússia e Turquemistão. ${ }^{3}$

$\mathrm{MnO}_{2}+2 \mathrm{KI}+2 \mathrm{H}_{2} \mathrm{SO}_{4} \longrightarrow \mathrm{Mn}^{2+}+\mathrm{I}_{2}+2 \mathrm{~K}^{+}+2 \mathrm{H}_{2} \mathrm{O}+2 \mathrm{SO}_{4}^{2-}$
$2 \mathrm{IO}_{3}^{-}+5 \mathrm{NaHSO}_{3} \longrightarrow \mathrm{I}_{2}+5 \mathrm{Na}^{+}+3 \mathrm{H}^{+}+5 \mathrm{SO}_{4}^{2-}+\mathrm{H}_{2} \mathrm{O}$

O iodo é um elemento indispensável à dieta, sendo o elemento mais pesado encontrado nos animais superiores. No final do século XIX, descobriu-se que o elemento é utilizado pela tireoide. O iodo participa da formação dos hormônios tireoidianos tri-iodotironina (T3) e tiroxina ou tetraiodotironina (T4), responsáveis por estimular o metabolismo celular. ${ }^{5}$ Muitos países (incluindo o Brasil) adotam o processo de adição de iodo ao sal de cozinha como forma de prevenir os males que a sua deficiência pode causar (bócio, cretinismo). ${ }^{3,5}$ Segundo a Resolução no 32 (25/02/2003) da Agência Nacional de Vigilância Sanitária (ANVISA), o sal deve conter entre 20 e 60 mg de iodo por $\mathrm{kg}$. A adição se dá na forma de iodato de potássio e não de iodeto, porque este último é oxidado pelo oxigênio atmosférico com o tempo. Pela mesma razão os frascos contendo iodeto devem ser conservados bem fechados em frascos escuros e ao abrigo da luz:

$4 \mathrm{XI}+\mathrm{O}_{2}+2 \mathrm{H}_{2} \mathrm{O} \longrightarrow 2 \mathrm{I}_{2}+4 \mathrm{XOH} \quad(\mathrm{X}=\mathrm{Na}, \mathrm{K})$

$\mathrm{O}$ aspecto amarelado dos reagentes antigos se deve à oxidação dos mesmos pelo oxigênio.

O iodo e seus compostos possuem uma ampla gama de aplicações, com destaque para a área médico-farmacêutica: antisséptico/desinfetante (tintura de iodo, polivinilpirrolidona); expectorante (solução de KI). ${ }^{6}$ Outra grande aplicação é na área analítica, para determinar o nível de insaturação de óleos e gorduras através do chamado "índice de iodo" (número de mg de $\mathrm{I}_{2}$ que reage com $1 \mathrm{~g}$ de amostra). Ele também é um dos reagentes do método para determinação de pequenas quantidades de água, especialmente em solventes orgânicos (método de Karl Fischer). ${ }^{7}$ O iodo, seja na forma elementar, de iodeto ou de periodato $\left(\mathrm{IO}_{4}^{-}\right)$, é empregado em análise e síntese orgânicas. ${ }^{8} \mathrm{KI}$ e $\mathrm{KIO}_{3}$ são utilizados em análise qualitativa (caracterização de amostras oxidantes ou redutoras, respectivamente) e também em análise quantitativa (titulação de oxirredução). ${ }^{7}$

O emprego de compostos de iodo em análise quantitativa começou pouco mais de 10 anos após a descoberta do elemento. Em 1826, Jacques Julien Houton de la Billardière (1755-1834) ferveu um pouco de 
iodo com solução de bicarbonato de sódio, amido de batata e cloreto de sódio, diluindo a solução resultante com água (1:1), empregando-a na análise de cal clorada. Neste procedimento forma-se iodeto de sódio, sendo oxidado pelo ânion hipoclorito $\left(\mathrm{OCl}^{-}\right)$a iodato; no ponto final da titulação o iodato e o iodeto reagem formando iodo, o qual em presença de amido confere à solução uma cor azul intensa. Esse método fez muito sucesso na época, sendo feitas várias tentativas de melhorar a prática experimental. ${ }^{9}$ Em 1840, M. Alphonse Du Pasquier foi o primeiro a propor o uso de iodo como reagente de titulação. Titulou uma solução de ácido sulfídrico $\left(\mathrm{H}_{2} \mathrm{~S}\right)$ com solução alcoólica de iodo, usando amido como indicador. Jöns Jacob Berzelius (1779-1848) achou este método interessante e inovador, mas qualificou-o de inexato porque o produto da redução do iodo, $\mathrm{HI}$ (iodeto de hidrogênio), reagia com iodo formando espécies mais complexas. Para evitar esse problema Berzelius propôs que se dissolvesse o iodo em solução aquosa de KI. ${ }^{9}$ Em 1845, Duflos determinou $\mathrm{Fe}^{3+}$ mediante adição de solução ácida de KI à amostra, e o iodo liberado foi titulado com solução de cloreto estanoso $\left(\mathrm{SnCl}_{2}\right) \cdot{ }^{9} \mathrm{No}$ ano seguinte, Henri-François Gaultier de Claubry (1792-1878, um dos descobridores da cor azul formada entre o iodo e o amido em $1814^{10}$ ) desenvolveu um método de análise de estanho por titulação com iodo. A sistematização dos métodos analíticos envolvendo iodo se deve a Robert Wilhelm Bunsen (1811-1899). ${ }^{11,12}$ Em 1853, ele generalizou a aplicabilidade do iodo para análise de substâncias oxidantes $\left[\mathrm{Br}_{2}, \mathrm{Cl}_{2}\right.$, $\left.\mathrm{ClO}_{3}{ }^{-}, \mathrm{IO}_{3}^{-}, \mathrm{Cr}(\mathrm{V}), \mathrm{Mn}(\mathrm{VII}), \mathrm{Ce}(\mathrm{IV}), \mathrm{Se}(\mathrm{VI}), \mathrm{V}(\mathrm{V}), \mathrm{Fe}(\mathrm{III}), \mathrm{As}(\mathrm{V})\right]$, mediante adição de solução de KI, seguida de titulação do iodo formado com solução aquosa de $\mathrm{SO}_{2}$. Esta última solução era de difícil preparação e era oxidada pelo ar com o tempo. Ainda em 1853, Karl Leonhard Heinrich Schwartz (1820-1890) recomendou a utilização de tiossulfato de sódio $\left(\mathrm{Na}_{2} \mathrm{~S}_{2} \mathrm{O}_{3}\right)$, mas seu uso suplantou o do $\mathrm{SO}_{2}$ somente cerca de 30 anos depois. Hoje, ele é praticamente o redutor universal do iodo. . $^{711}$

Os métodos envolvendo iodo são classificados em dois grupos:? quando um analito redutor é titulado diretamente com iodo (produzindo íons iodeto), o método é denominado iodimetria. Caso o analito seja oxidante, ele é adicionado a um excesso de íons iodeto, produzindo iodo (ou melhor, tri-iodeto, $\mathrm{I}_{3}^{-}$), o qual é titulado com solução de tiossulfato. O método é denominado iodometria.

O iodo não é muito solúvel em água (a solução saturada contém $\left.0,0013 \mathrm{~mol} \mathrm{~L}^{-1}\right)$. A solubilidade aumenta adicionando-se iodo elementar a uma solução de KI. O iodo reage com o íon iodeto produzindo íons tri-iodeto:

$$
\mathrm{I}_{2}+\mathrm{I}^{-} \rightleftarrows \mathrm{I}_{3}^{-} \quad\left(\mathrm{K}_{\text {form }}=7,4 \times 10^{2}\right)
$$

Outro método é reagir iodato de potássio $\left(\mathrm{KIO}_{3}\right.$, padrão primário) com leve excesso de KI em solução aquosa acidificada por ácido mineral forte $(\mathrm{pH} \sim 1):^{13}$

$$
\mathrm{IO}_{3}^{-}+8 \mathrm{I}^{-}+6 \mathrm{H}^{+} \longrightarrow 3 \mathrm{I}_{3}^{-}+3 \mathrm{H}_{2} \mathrm{O}
$$

A reação do íon tri-iodeto com o íon tiossulfato em meio ácido a neutro produz íons iodeto e tetrationato: ${ }^{7}$

$$
\mathrm{I}_{3}^{-}+2 \mathrm{~S}_{2} \mathrm{O}_{3}{ }^{2-} \longrightarrow 3 \mathrm{I}^{-}+\mathrm{S}_{4} \mathrm{O}_{6}{ }^{2-}
$$

Ela é mais bem conduzida em $\mathrm{pH}$ abaixo de 9 para evitar o desproprocionamento de $\mathrm{I}_{3}^{-}$em I- e $\mathrm{IO}^{-}$(hipoiodito). Este último oxida $\mathrm{S}_{2} \mathrm{O}_{3}{ }^{2-}$ a sulfato $\left(\mathrm{SO}_{4}{ }^{2-}\right)$. Embora o íon tiossulfato se decomponha em íons hidrogenossulfito $\left(\mathrm{HSO}_{3}{ }^{-}\right)$e enxofre elementar em meio ácido, a oxidação do mesmo pelo íon $\mathrm{I}_{3}$ - é cineticamente mais rápida que sua decomposição. $\mathrm{O}$ amido permite um limite de detecção 10 vezes menor do que apenas com a coloração dos íons $\mathrm{I}_{3}$.

Merece também destaque o emprego do chamado reagente de

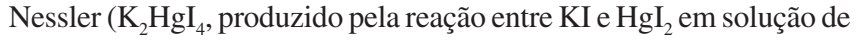
$\mathrm{KOH})$ para detecção e quantificação de nitrogênio amoniacal $\left(\mathrm{NH}_{4}{ }^{+}\right){ }^{14}$

Nas primeiras décadas do século XX, houve uma preocupação em recuperar o iodo dos resíduos laboratoriais. A razão para isso era o alto custo de seus reagentes, considerados "dos mais caros de um laboratório analítico". ${ }^{15}$ Por isso, diversos procedimentos foram desenvolvidos para recuperar o iodo de seus resíduos, os quais são adaptações dos métodos empregados para obtenção do elemento de fontes naturais. ${ }^{16,17} \mathrm{~A}$ oxidação do íon iodeto remanescente é feita em meio ácido por $\mathrm{MnO}_{2}$, íons $\mathrm{IO}_{3}^{-} \mathrm{e}$, particularmente, $\mathrm{Cl}_{2}$. A acidez não deve ser elevada para evitar que o iodo seja parcialmente oxidado a iodato. O iodo bruto é filtrado; para purificá-lo, uma alternativa frequente é reduzir o iodo bruto com solução aquosa de $\mathrm{SO}_{2}$ ou de $\mathrm{NaHSO}_{3}$ em meio fracamente ácido, seguido de filtração das impurezas e reoxidação do iodeto. ${ }^{13,18,19}$

Outro composto de iodo empregado em laboratório é a solução de HI (ácido iodídrico) em sínteses orgânicas (por exemplo, conversão de ácidos carboxílicos a hidrocarbonetos; conversão de alcoóis primários a haletos de alquila; clivagem de éters em iodetos de alquila e alcoóis) e inorgânicas (como fonte de iodo e agente redutor). O HI é um reagente caro, sendo vendido em frascos âmbar, visto que pode ser lentamente oxidado pelo oxigênio atmosférico. A oxidação é acelerada pela luz: ${ }^{20}$

$$
\begin{gathered}
4 \mathrm{HI}+\mathrm{O}_{2} \longrightarrow 2 \mathrm{H}_{2} \mathrm{O}+2 \mathrm{I}_{2} \\
\mathrm{HI}+\mathrm{I}_{2} \rightleftarrows \mathrm{HI}_{3}
\end{gathered}
$$

A solução saturada contém $70 \% \mathrm{~m} / \mathrm{m}$ de HI. O produto comercial normalmente é um azeótropo contendo $57 \% \mathrm{~m} / \mathrm{m}$ de $\mathrm{HI}(\mathrm{d}=1,70 \mathrm{~g}$ $\left.\mathrm{mL}^{-1}, \mathrm{p}_{\mathrm{eb}}=127^{\circ} \mathrm{C}\right)$. É um dos ácidos minerais mais fortes $\left(K_{\mathrm{a}} \sim 10^{10}\right){ }^{7}$ É preparado industrialmente pela reação de iodo com hidrazina: ${ }^{3}$

$$
2 \mathrm{I}_{2}+\mathrm{N}_{2} \mathrm{H}_{4} \longrightarrow 4 \mathrm{HI}+\mathrm{N}_{2}
$$

No laboratório, é preparado pela reação entre iodo e fósforo vermelho (ou ácido hipofosforoso ou ácido fosforoso) em água, seguida de aquecimento para se obter HI azeotrópico: $:^{7,20}$

$$
3 \mathrm{I}_{2}+2 \mathrm{P}+6 \mathrm{H}_{2} \mathrm{O} \longrightarrow 6 \mathrm{HI}+2 \mathrm{H}_{3} \mathrm{PO}_{3}
$$

Ao contrário do que sucede com o iodo e o íon iodeto, poucos trabalhos relatam a recuperação de $\mathrm{HI}$ de seus resíduos; ${ }^{4}$ a rota é a redução do elemento a HI, seguida de destilação do azeótropo.

$\mathrm{Na}$ literatura nacional existem vários trabalhos que focam a recuperação do bromo de seus resíduos laboratoriais. ${ }^{21} \mathrm{~A}$ menção ao iodo é mais escassa; ${ }^{22}$ os métodos relatados são de natureza oxidativa. Embora soluções diluídas contendo iodo possam ser descartadas na forma de íons iodeto, ${ }^{23}$ os resíduos com quantidades consideráveis desse elemento devem ser geridos sob o ponto de vista da recuperação do mesmo, pois em caso de descarte os impactos ambientais são inevitáveis. ${ }^{23}$ Este trabalho visa estudar o tratamento de reagentes e soluções deteriorados de iodetos, com vistas a obter o iodo na forma elementar ou de um composto utilizável em práticas laboratoriais.

\section{PARTE EXPERIMENTAL}

\section{Resíduos e reagentes contendo iodo}

Foram empregados 7 frascos de ácido iodídrico azeotrópico. Quatro deles contêm $250 \mathrm{~mL}$ do produto, são de origem alemã (Riedel) e foram fabricados em 1975; os outros 3 frascos contêm $100 \mathrm{~mL}$, sendo um de origem norte-americana (Mallinckrodt) e dois de origem alemã (Merck), fabricados, respectivamente, em 1943 e 1970. Apesar do vidro âmbar e da dupla vedação da tampa rosqueada, todas as soluções apresentavam coloração castanha escura. 
Foram processados 3 frascos de iodeto de potássio, abertos diversas vezes para coleta do sal. Todos eles são de procedência nacional (Vetec) e foram fabricados em 2000. Além da presença de água (o KI é higroscópico), a solução e o sólido tinham coloração amareloalaranjada face à oxidação parcial do íon iodeto pelo oxigênio. Duas soluções (250 mL cada) de KI 0,3 mol L-1 de coloração amarelada, foram também utilizadas.

A manipulação desses produtos exige luvas, óculos e jaleco, porque o iodo é rapidamente absorvido pela pele e seu vapor é muito irritante para mucosas e olhos. O ambiente de trabalho deve ser ventilado, por isso todos os experimentos foram feitos em uma capela (a concentração máxima admitida de iodo no ambiente de trabalho é 0,1 $\left.\mathrm{mg} \mathrm{m}^{-3}\right){ }^{24}$ Nas pesagens, empregou-se uma balança comum, pois os vapores de iodo danificam a balança analítica. Durante o manuseio, os frascos devem ser protegidos da incidência de luz direta, evitando a aceleração da oxidação do íon iodeto. Manteve-se uma solução de $\mathrm{Na}_{2} \mathrm{~S}_{2} \mathrm{O}_{3}$ de aproximadamente $0,1 \mathrm{~mol} \mathrm{~L}^{-1}$ para neutralizar eventuais derramamentos de solução de HI ou de KI na capela ou mesmo sobre as luvas (o iodo é imediatamente reduzido a iodeto).

\section{Recuperação do iodo por via oxidativa}

Esta rota se baseia na reação com peróxido de hidrogênio segundo as reações:

$$
\begin{gathered}
2 \mathrm{HI}+\mathrm{H}_{2} \mathrm{O}_{2} \longrightarrow \mathrm{I}_{2}+2 \mathrm{H}_{2} \mathrm{O} \\
2 \mathrm{KI}+\mathrm{H}_{2} \mathrm{O}_{2} \longrightarrow \mathrm{I}_{2}+2 \mathrm{KOH}
\end{gathered}
$$

$250 \mathrm{~mL}$ da solução de HI foram colocados em um béquer de $1000 \mathrm{~mL}$, que foi colocado em uma cuba contendo gelo. Devido ao fato de a reação entre $\mathrm{HI}$ (ou $\mathrm{KI}$ ) e $\mathrm{H}_{2} \mathrm{O}_{2}$ ser muito exotérmica (como constatado em testes em tubo de ensaio), o resfriamento se fazia necessário para evitar que parte do iodo sublimasse com o calor desprendido no processo de oxidação. A cuba foi colocada sobre uma placa agitadora. Após o início da agitação magnética da solução de HI (200 rpm), adicionou-se, lentamente, solução de $\mathrm{H}_{2} \mathrm{O}_{2}$ a $30 \% \mathrm{~m} / \mathrm{m}\left(4 \mathrm{~mL} \mathrm{~min}^{-1}\right)$. O iodo formado se depositava no fundo do béquer, enquanto que a solução mantinha uma coloração alaranjada. A adição cessou quando não havia mais deposição de iodo. É importante evitar excesso de oxidante, pois, devido ao meio ácido e ao forte poder oxidante do reagente, parte do iodo pode ser oxidada a ácido iódico $\left(\mathrm{HIO}_{3}\right)$ :

$$
\mathrm{I}_{2}+5 \mathrm{H}_{2} \mathrm{O}_{2} \longrightarrow 2 \mathrm{HIO}_{3}+4 \mathrm{H}_{2} \mathrm{O}
$$

A solução foi deixada em repouso por $4 \mathrm{~h}$ (sob gelo) para que todo o iodo se assentasse no fundo do béquer. $\mathrm{O}$ sobrenadante tinha cor alaranjada. Em seguida, filtrou-se o iodo em funil de Büchner a vácuo (empregou-se papel de filtro de filtração lenta). Para proteger a bomba de eventuais vapores de iodo sublimados, o frasco de guarda estava imerso em gelo seco e acetona e o kitazato coletor do filtrado estava em banho de gelo. É aconselhável mexer ocasionalmente a massa de iodo com um bastão de vidro para liberar eventuais porções de água enclausuradas na mesma. O iodo foi colocado em um frasco âmbar com tampa rosqueada, o qual foi pesado antes e depois do procedimento para se estimar a massa do elemento recuperada.

O filtrado alaranjado foi recolocado no béquer e tratado com mais um pouco de solução de $\mathrm{H}_{2} \mathrm{O}_{2}$. Ele descorou e a solução tinha $\mathrm{pH}$ ácido (em torno de 2, avaliado com papel indicador universal). Essa solução foi evaporada lentamente $\left(50-60^{\circ} \mathrm{C}\right)$ na capela, obtendo-se um sólido branco (ácido iódico), que foi pesado e guardado em frasco escuro.

$\mathrm{O}$ procedimento para o KI é uma adaptação do processo para o HI. A diferença se deve à formação de $\mathrm{KOH}$ como um dos produtos da oxidação do sal; o aumento da concentração da base forte no meio reacional eleva o risco de desproporcionamento do iodo:

$$
\mathrm{I}_{2}+2 \mathrm{KOH} \longrightarrow \mathrm{KI}+\mathrm{KIO}+\mathrm{H}_{2} \mathrm{O}
$$

O íon hipoiodito se decompõe rapidamente:

$$
3 \mathrm{IO}^{-} \longrightarrow 2 \mathrm{I}^{-}+\mathrm{IO}_{3}^{-}
$$

de modo que a reação global é

$$
3 \mathrm{I}_{2}+6 \mathrm{KOH} \longrightarrow 5 \mathrm{KI}+\mathrm{KIO}_{3}+3 \mathrm{H}_{2} \mathrm{O}
$$

Em média, de cada frasco de KI, foram separados $30 \mathrm{~g}$ do sal e do líquido sobrenadante, que foram dissolvidos em $250 \mathrm{~mL}$ de água. Todas as soluções de KI foram processadas da mesma forma como no caso do $\mathrm{HI}$, tendo-se o cuidado de adicionar periodicamente $\mathrm{H}_{2} \mathrm{SO}_{4}$ $1 \mathrm{~mol} \mathrm{~L}^{-1}$ para manter o pH da solução abaixo de 9 .

\section{Recuperação de compostos de iodo por via redutora}

Para reduzir o iodo presente nas soluções de $\mathrm{HI}\left(\mathrm{HI}_{3}\right)$, empregouse fósforo vermelho, insumo disponível no laboratório. O manuseio desse reagente exige os mesmos cuidados dos compostos de iodo e uma máscara para pós. As reações são:

$$
\begin{gathered}
2 \mathrm{P}+3 \mathrm{HI}_{3} \longrightarrow 2 \mathrm{PI}_{3}+3 \mathrm{HI} \\
\mathrm{PI}_{3}+3 \mathrm{H}_{2} \mathrm{O} \longrightarrow \mathrm{H}_{3} \mathrm{PO}_{3}+3 \mathrm{HI}
\end{gathered}
$$

Em aparelhagem para destilação simples, colocaram-se $5 \mathrm{~g}$ de fósforo vermelho (pesados em balança comum) em um balão de fundo redondo $(500 \mathrm{~mL})$ e $250 \mathrm{~mL}$ de solução de $\mathrm{HI}$ a ser tratada. O conjunto reacional foi aquecido por meio de uma manta aquecedora a $70-80{ }^{\circ} \mathrm{C}$ por $1 \mathrm{~h}$, a fim de que o iodo fosse reduzido pelo fósforo vermelho, atestado pelo clareamento da solução, até se tornar incolor. Após esse período, elevou-se a temperatura da manta para permitir a destilação do HI azeotrópico $\left(127^{\circ} \mathrm{C}\right)$. O condensador e o frasco coletor (que é do próprio reagente, limpo e seco) estavam cobertos por papel alumínio e o frasco estava imerso em banho de gelo. É importante verificar o estado das conexões da aparelhagem para que não haja admissão de ar no interior da mesma, com consequente oxidação do HI. Após a destilação (o destilado deve ser límpido e incolor), o frasco coletor é imediatamente retirado e tampado, sendo conservado ao abrigo da luz direta.

Novas porções de $250 \mathrm{~mL}$ de $\mathrm{HI}$ podem ser adicionadas ao balão e o processo de redução é repetido. Ao final dos tratamentos, a massa viscosa contendo fósforo vermelho residual e um sólido esbranquiçado foi tratada com solução de $\mathrm{NaOH} 6 \mathrm{~mol} \mathrm{~L}^{-1}$. O fósforo vermelho foi isolado por filtração e a solução alcalina, incolor, foi submetida a testes qualitativos para se determinar a natureza dos produtos de oxidação presentes, contendo fósforo..$^{25,26}$

Esse método não se aplica ao caso do KI, pois obter-se-ía uma mistura de produtos de difícil separação:

$$
\begin{gathered}
3 \mathrm{KI}_{3}+2 \mathrm{P}+6 \mathrm{H}_{2} \mathrm{O} \longrightarrow 3 \mathrm{KI}+2 \mathrm{H}_{3} \mathrm{PO}_{3}+6 \mathrm{HI} \\
3 \mathrm{KOH}+\mathrm{HI}+\mathrm{H}_{3} \mathrm{PO}_{3} \longrightarrow \mathrm{KI}+\mathrm{K}_{2} \mathrm{HPO}_{3}+3 \mathrm{H}_{2} \mathrm{O}
\end{gathered}
$$

O método empregado é uma adaptação da síntese industrial do HI (descrita na reação 9). Em um béquer, $250 \mathrm{~mL}$ de solução contendo $\mathrm{KI}\left(\mathrm{KI}_{3}\right.$ ) foram misturados a $15 \mathrm{~mL}$ de hidrazina $1 \mathrm{~mol} \mathrm{~L}^{-1}$ (a manipulação dessa solução é feita da mesma forma que os compostos de iodo). A mistura reacional foi aquecida a $30-40^{\circ} \mathrm{C}$. Em $1 \mathrm{~h}$, a solução descorou totalmente e a liberação de gás $\left(\mathrm{N}_{2}\right)$ cessou. $\mathrm{O} \mathrm{HI}$ gerado 
in situ reage com o KOH produzido na oxidação do KI (reação 3), regenerando esse sal em solução.

\section{Métodos analíticos}

As soluções de $\mathrm{HI}$ (ou de $\mathrm{KI}$ ) contendo iodo foram tituladas com $\mathrm{Na}_{2} \mathrm{~S}_{2} \mathrm{O}_{3} 0,095 \mathrm{~mol} \mathrm{~L}^{-1}$ usando amido como indicador ${ }^{27}$ para avaliar o percentual de iodo oxidado nesses reagentes. Alíquotas das soluções de $\mathrm{HI}$ ou $\mathrm{KI}$ obtidas após a rota redutora foram adicionadas à solução de $\mathrm{K}_{2} \mathrm{Cr}_{2} \mathrm{O}_{7} 0,054 \mathrm{~mol} \mathrm{~L}^{-1}\left(\mathrm{em} \mathrm{H}_{2} \mathrm{SO}_{4} 0,5 \mathrm{~mol} \mathrm{~L}^{-1}\right)$ e o tri-iodeto obtido foi titulado com solução de $\mathrm{Na}_{2} \mathrm{~S}_{2} \mathrm{O}_{3} 0,095 \mathrm{~mol} \mathrm{~L}^{-1}$ usando amido como indicador. ${ }^{27} \mathrm{~A}$ densidade do $\mathrm{HI}$ azeotrópico destilado foi obtida por meio de um densímetro (acidímetro) calibrado para soluções desse ácido. $\mathrm{O}$ iodo obtido pela rota oxidante foi apenas avaliado quanto à massa obtida. O ácido iódico obtido como subproduto desta rota foi dissolvido em água e caracterizado por meio de ensaios qualitativos. ${ }^{25,26}$

\section{RESULTADOS E DISCUSSÃO}

\section{Recuperação do iodo pela via oxidativa}

Com base nas concentrações nominais de $\mathrm{HI}(57 \% \mathrm{~m} / \mathrm{m}, \mathrm{d}=1,7$ $\left.\mathrm{g} \mathrm{mL}^{-1},[\mathrm{HI}]=7,57 \mathrm{~mol} \mathrm{~L}^{-1}\right)$ e de $\mathrm{H}_{2} \mathrm{O}_{2}\left(30 \% \mathrm{~m} / \mathrm{m}, \mathrm{d}=1,1 \mathrm{~g} \mathrm{~mL}^{-1}\right.$, $\left[\mathrm{H}_{2} \mathrm{O}_{2}\right]=9,7 \mathrm{~mol} \mathrm{~L}^{-1}$ ), previa-se que, para cada $250 \mathrm{~mL}$ de $\mathrm{HI}$ azeotrópico, eram necessários cerca de $200 \mathrm{~mL}$ de $\mathrm{H}_{2} \mathrm{O}_{2}$ a $30 \% \mathrm{~m} / \mathrm{m}$. Em média, o volume empregado foi de $190 \mathrm{~mL}$, portanto abaixo do teórico esperado, explicável pela presença de iodo pré-oxidado antes do tratamento. Esse ponto foi esclarecido quando da titulação da solução original com tiossulfato de sódio. As soluções de HI fabricadas na década de 1970 tinham cerca de $6 \%$ do HI original oxidado, enquanto que a amostra fabricada na década de 1940 tinha uma fração oxidada bem maior, $15 \%$.

Um aspecto muito importante da rota oxidativa é evitar excesso de $\mathrm{H}_{2} \mathrm{O}_{2}$, o que justifica a adição lenta desse reagente e a agitação intensa da mistura reacional (sob resfriamento). Isso tem dois efeitos: evitar a geração demasiada de calor, fazendo com que parte do iodo escape da solução e, evitar oxidação local do iodo a ácido iódico. Nas nossas condições experimentais, a adição da solução de $\mathrm{H}_{2} \mathrm{O}_{2}$ levou pouco menos de $1 \mathrm{~h}\left(4 \mathrm{~mL} \mathrm{~min}^{-1}\right)$. O repouso tinha a finalidade de permitir a decantação das partículas mais finas do elemento. Face à sua densidade $\left(4,93 \mathrm{~g} \mathrm{~cm}^{-3}\right)$ e à ausência de excesso de $\mathrm{H}_{2} \mathrm{O}_{2}(\mathrm{o}$ que poderia levar à flotação das partículas mais finas por conta do oxigênio desprendido na decomposição do peróxido), em 4 h esse objetivo foi alcançado.

Para cada $250 \mathrm{~mL}$ de HI processado, obteve-se em média 233 g de $\mathrm{I}_{2}$, o que dá um rendimento de $97 \%$ em massa em relação ao teórico (240,2 g). Existem duas fontes de perda de iodo: a sublimação de parte deste no momento do isolamento (particularmente na etapa de filtração a vácuo, comprovada pela presença de coloração amarelada no frasco de guarda imerso em gelo seco e acetona) e, a perda do elemento devido à sua solubilidade (mesmo que limitada) em água. Esse último problema pode ser minimizado pelo emprego de solução mais concentrada de $\mathrm{H}_{2} \mathrm{O}_{2}$ a fim de reduzir o volume da mistura reacional. Essa perda parece ser o fator principal, porque a massa média do $\mathrm{HIO}_{3}$ isolado após o tratamento da solução aquosa contendo iodo residual com $\mathrm{H}_{2} \mathrm{O}_{2}$ foi de $7 \mathrm{~g}$, correspondendo a $4,7 \mathrm{~g}$ de iodo (quase $2 \%$ do iodo total inicial). Como o ácido iódico é um ácido forte $\left(K_{\mathrm{a}}=0,16 \mathrm{~mol} \mathrm{~L}^{-1}\right),{ }^{28}$ isso justifica o $\mathrm{pH}$ da solução obtido quando da oxidação do iodo pelo $\mathrm{H}_{2} \mathrm{O}_{2}$. Cabe ainda salientar que a obtenção do ácido iódico somente foi efetiva quando a solução era evaporada lentamente, pois uma evaporação mais intensa (sob ebulição) levava à decomposição parcial do composto com produção de vapores de iodo. Este composto foi dissolvido em água para preparar uma solução de aproximadamente $0,1 \mathrm{~mol} \mathrm{~L}^{-1}$, empregada em Química Analítica Qualitativa na análise dos ânions do grupo volátil..$^{26,27}$

Com base nos preços médios ${ }^{29}$ da solução de $\mathrm{H}_{2} \mathrm{O}_{2}$ a $30 \% \mathrm{~m} / \mathrm{m}$ (1 $\mathrm{L}-\mathrm{R} \$ 160,00)$ e do $\mathrm{I}_{2}(500 \mathrm{~g}-\mathrm{R} \$ 128,00)$, o consumo de $190 \mathrm{~mL}$ da solução do oxidante $(\mathrm{R} \$ 30,40)$ produziu $233 \mathrm{~g}$ do produto $(\mathrm{R} \$$ 59,65). Nesse aspecto, o balanço econômico é favorável à recuperação do iodo de seus resíduos de HI por via oxidativa.

O processamento das soluções oriundas do reagente KI foi muito similar ao das soluções de HI. Cerca de $15 \%$ do iodo estava préoxidado. A concentração de KI nas soluções preparadas a partir dos reagentes deteriorados era em torno de $0,6 \mathrm{~mol} \mathrm{~L}^{-1} .250 \mathrm{~mL}$ dessa solução consumiram $6,7 \mathrm{~mL}$ de solução de $\mathrm{H}_{2} \mathrm{O}_{2}$ a $30 \% \mathrm{~m} / \mathrm{m}(\mathrm{R} \$$ 1,07), enquanto que $250 \mathrm{~mL}$ da solução de $\mathrm{KI} 0,3 \mathrm{~mol} \mathrm{~L}^{-1}$ consumiram $3,3 \mathrm{~mL}(\mathrm{R} \$ 0,53)$. A massa de iodo recuperada de cada solução foi, respectivamente, 18,29 $\mathrm{g}(\mathrm{R} \$ 4,68)$ e $9,14 \mathrm{~g}(\mathrm{R} \$ 2,34)$. O rendimento foi de $96 \%$ em relação ao iodo total inicialmente presente em todos os casos. Como no caso do HI, com base nos preços dos reagentes e dos produtos, a recuperação do iodo é factível. Cerca de $2 / 3$ das perdas são devidas à formação de $\mathrm{KIO}_{3}$ a partir do iodo remanescente no filtrado. O resíduo final é uma solução incolor, de $\mathrm{pH}$ aproximadamente 7, contendo $\mathrm{K}_{2} \mathrm{SO}_{4}$ e $\mathrm{KIO}_{3}$. Para separá-los, adicionou-se, gota a gota, solução de $\mathrm{Ca}\left(\mathrm{NO}_{3}\right)_{2}, 0,1 \mathrm{~mol} \mathrm{~L}^{-1}$ sob aquecimento (60 $\left.{ }^{\circ} \mathrm{C}\right)$. O sal $\mathrm{Ca}\left(\mathrm{IO}_{3}\right)_{2}$ precipitou sem que houvesse coprecipitação do $\mathrm{CaSO}_{4}$, mais solúvel em água quente do que o iodato. ${ }^{28}$

\section{Recuperação de HI e KI pela via redutora}

Para cada $100 \mathrm{~mL}$ de solução original de HI obteve-se um volume médio de 99,5 mL de destilado, indicando que a recuperação do iodo (como HI) pela via redutora apresentou rendimento um pouco maior do que pela via oxidativa. Além do aspecto incolor do destilado, sua densidade corresponde àquela do HI azeotrópico. Portanto, corroborando estudos prévios da literatura, ${ }^{4}$ é possível recuperar este reagente dispendioso a partir do mesmo deteriorado. Da mesma forma, o reagente KI foi recuperado com um rendimento maior $(99,8 \% \mathrm{~m} / \mathrm{m})$ do que o iodo obtido pela via oxidativa $(96 \% \mathrm{~m} / \mathrm{m})$.

A razão para este comportamento tem origem na forma como as soluções originais foram processadas. Enquanto há necessidade de uma etapa de separação sólido-líquido (isolamento do iodo) na rota oxidativa, com consequente perda de iodo face à sua solubilidade em água, a via redutora mantém o iodo integralmente na solução original (a destilação do HI azeotrópico separou-o do fósforo vermelho e dos produtos de oxidação deste - sólidos - adicionados à solução original). No caso do KI, o emprego da hidrazina tem a vantagem de não gerar resíduos na solução final, evitando etapas de separação.

$5 \mathrm{~g}$ de fósforo vermelho trataram 4 porções de $250 \mathrm{~mL}$ de HI, obtendo-se $995 \mathrm{~mL}$ do produto. $1 \mathrm{~L}$ de $\mathrm{HI}$ azeotrópico custa em média $\mathrm{R} \$ 1.698,00 .^{29} 5 \mathrm{~g}$ de fósforo vermelho custam cerca de $\mathrm{R} \$ 2,90 .{ }^{29}$ Devido ao alto valor agregado do produto obtido e ao baixo custo do reagente redutor (o fósforo vermelho é mais barato do que os ácidos hipofosforoso e fosforoso ${ }^{29}$ ), mesmo não tendo sido levado em conta o consumo energético, o balanço econômico será favorável. O tratamento das soluções deterioradas de KI com solução de $\mathrm{N}_{2} \mathrm{H}_{4}$ também segue essa mesma tendência. $250 \mathrm{~g}$ de KI custam em média $\mathrm{R} \$ 86,00 .{ }^{29}$ Assim, $250 \mathrm{~mL}$ de solução $0,3 \mathrm{~mol}$ $\mathrm{L}^{-1}$ de KI contêm 12,45 g do sal $(\mathrm{R} \$ 4,29)$ e $250 \mathrm{~mL}$ de uma solução $0,6 \mathrm{~mol} \mathrm{~L}^{-1}$ contêm $24,90 \mathrm{~g}(\mathrm{R} \$ 8,57) .1 \mathrm{~L}$ de $\mathrm{N}_{2} \mathrm{H}_{4}$ comercial $(24 \% \mathrm{~m} / \mathrm{m})$ contém cerca de $240 \mathrm{~g}$ de $\mathrm{N}_{2} \mathrm{H}_{4}\left(7,5 \mathrm{~mol} \mathrm{~L}^{-1}\right)$ e custa $\mathrm{R} \$ 50,00 .{ }^{29} 15 \mathrm{~mL}$ de uma solução $1 \mathrm{~mol} \mathrm{~L}^{-1}$, diluída a partir de 2 $\mathrm{mL}$ do produto comercial, equivalem a apenas $\mathrm{R} \$ 0,10$. O balanço econômico é mais favorável para as rotas redutoras do que para a via oxidativa, por conta do custo mais elevado do $\mathrm{H}_{2} \mathrm{O}_{2}$ frente ao 
fósforo vermelho e à hidrazina e do preço mais elevado do HI e do $\mathrm{KI}$ frente ao iodo elementar.

$\mathrm{O}$ pH da solução final de KI obtida (incolor) estava entre 6 e 7. As soluções de KI empregadas normalmente nas práticas laboratoriais do IQ/UFRJ têm concentração variando de 0,1 a $0,3 \mathrm{~mol} \mathrm{~L}^{-1}$, de modo que o produto obtido pode ser usado após diluição conveniente em tais práticas (Análise Qualitativa: ${ }^{26,27}$ determinação do caráter oxidante de soluções aquosas, precipitação de iodetos; Análise Quantitativa:25,28 práticas de iodometria; Análise Orgânica: ${ }^{30}$ análise qualitativa de derivados halogenados; Química Orgânica Experimental: ${ }^{30}$ clivagem de éteres, síntese de iodobenzeno; Química Geral Experimental:26,28 preparação de iodo, estudo de reações redox).

A análise qualitativa dos produtos de oxidação do fósforo vermelho (isolados quando do tratamento da massa sólida do balão de destilação com solução de $\mathrm{NaOH}$ ) indica que são fortemente redutores. A adição de sais solúveis de $\mathrm{Ca}^{2+}$ e $\mathrm{Ba}^{2+}$ levou à precipitação de sólidos brancos; com íons $\mathrm{Ag}^{+}$formou-se um precipitado branco, que paulatinamente se tornou preto (prata metálica). Com base nesses resultados, o produto de oxidação formado foi provavelmente o ácido fosforoso $\left(\mathrm{H}_{3} \mathrm{PO}_{3}\right)$, uma das possibilidades aventadas na literatura quando da reação do $\mathrm{I}_{2}$ com fósforo vermelho em presença de água. ${ }^{4}$ Este é o único resíduo final de processo da via redutora. Em média, para cada $10 \mathrm{~mL}$ de solução de $\mathrm{HI}$ processado, obteve-se $0,1 \mathrm{~mL}$ de solução final. Ela pode ser oxidada com $\mathrm{H}_{2} \mathrm{O}_{2}$ (com produção de ácido fosfórico, $\left.\mathrm{H}_{3} \mathrm{PO}_{4}\right),{ }^{31} \mathrm{o}$ qual pode ser utilizado para precipitar metais de transição de resíduos oriundos de aulas experimentais.

\section{CONCLUSÕES}

Para a gestão de resíduos contendo quantidades importantes de iodo, as rotas oxidativa e redutora apresentaram resultados muito bons quanto à recuperação do elemento ( $\mathrm{I}_{2}, \mathrm{KI}$ ou $\left.\mathrm{HI}\right)$, sob condições experimentais acessíveis à rotina laboratorial. Face à ausência de etapas de separação sólido-líquido, os rendimentos da via redutora foram um pouco maiores; na rota oxidativa, o subproduto obtido foi o ácido iódico oriundo das perdas do iodo solubilizado em água. Os resíduos finais de processo gerados correspondem a uma fração mínima da quantidade de material inicialmente processado.

Além dos aspectos relativos à gestão eficiente dos resíduos (os quais foram convertidos em insumos reutilizáveis), este trabalho se constituiu num excelente exercício da aplicação de diversas técnicas de laboratório (filtração, destilação, pesagem, determinação de densidade, avaliação do pH, titulações redox etc.) e de conscientização quanto aos cuidados com a higiene e a saúde ocupacionais no ambiente de trabalho. Este trabalho pode ser adaptado a uma aula prática, por exemplo, de Química Geral Experimental, de 4 h de duração.

\section{MATERIAL SUPLEMENTAR}

Disponível gratuitamente na forma de arquivo pdf em http:// www.quimicanova/sbq.org.br, apresenta o aspecto de uma solução de ácido iodídrico antes e depois do tratamento da mesma com fósforo vermelho, seguido de destilação do azeótropo.

\section{AGRADECIMENTOS}

Ao CNPq pelo auxílio financeiro.

\section{REFERÊNCIAS E NOTAS}

1. Weeks, M. E.; Discovery of the elements, $5^{\text {th }}$ ed., Journal of Chemical Education: Easton, 1945, p. 442-449.

2. Greenwood, N. N.; Earnshaw, A.; Chemistry of the Elements, $2^{\text {nd }}$ ed., Butterworth-Heinemann: Oxford, 1997, p. 809-815.

3. The United States Geological Survey; Mineral Commodity Summaries: Reston, January 2011, p. 76-77.

4. Robinson, J. M.; Herndon, P. T.; Holland, P. L.; Marrufo, L. D.; Org. Proc. Res. Dev. 1999, 3, 352.

5. Fuge, R.; Soils and Iodine Deficiency. Essentials of Medical Geology, Elsevier: Amsterdam, 2005, p. 417-433.

6. Gottardi, W.; Sterilants, Disinfectants and Antiseptics. Disinfection, Sterilization and Preservation, $5^{\text {th }}$ ed., Lippincot Williams \& Wilkins: Philadelphia, 2001, p. 159-183.

7. Harris, D. C.; Análise Química Quantitativa, tradução da $7^{\mathrm{a}}$ ed., LTC: Rio de Janeiro, 2008, p. 375-378.

8. Bruice, P. Y.; Organic Chemistry, $4^{\text {th }}$ ed., Prentice Hall: Upper Saddle River, 2003, p. 438-439, 452.

9. Szabadráry, F.; History of Analytical Chemistry, Gordon and Breach Science Publishers: Langhorne, 1992, p. 228-230.

10. Popoff, S.; Whitman, J. L.; J. Am. Chem. Soc. 1925, 47, 2259.

11. Szabadráry, F.; Chalmers, R. A.; Talanta 1979, 26, 609.

12. Xie, Y.; McDonald, M. R.; Margerum, D. W.; Inorg. Chem. 1999, 38, 3938.

13. De Witt, C. C.; J. Chem. Educ. 1937, 14, 215.

14. Wirth, H. E.; Robinson, R. J.; Ind. Eng. Chem. Anal. Ed. 1933, 5, 293.

15. Bonner, W. D.; Masaki, K.; J. Chem. Educ. 1930, 7, 616.

16. White, C. E.; J. Chem. Educ. 1935, 12, 169; Wahab, M. F.; J. Chem. Educ. 2009, 86, 206.

17. Smith, A.; Ind. Eng. Chem. 1914, 6, 83.

18. Rosenfeld, L.; J. Chem. Educ. 2000, 77, 984.

19. Schimpuff, G.; Pottinger, R.; Ind. Eng. Chem. Anal. Ed. 1941, 13, 337.

20. Ohlweiler, O. A.; Química Inorgânica, Edgard Blücher e Editora da Universidade de São Paulo: São Paulo, 1971, vol. I, p. 57-58.

21. Tavares, G. A.; Bendassolli, J. A.; Souza, G.; Nolasco, F. R.; Bonassi, J. A.; Batagello, H. H.; Quim. Nova 2004, 27, 320.

22. Gerbase, A. E.; Gregório, J. R.; Calvete, T.; Quim. Nova 2006, $29,397$.

23. On-Site Water Purification and Inactivation of E. coli and MS-2 Virus in Raw Drinking Water, Environmental Technology Verification Report, NSF International and The US Environmental Protection Agency: Cincinnati, 2001.

24. http://www.osha.gov/dts/chemicalsampling/data/CH_247201.html, acessada em Fevereiro 2011.

25. Krauledat, W. G.; Práticas de Análise Volumétrica, série B-1, Fundação Técnico-Educacional Souza Marques: Rio de Janeiro, 1979, p. 64-67.

26. Vogel, A. I.; Química Analítica Qualitativa, 5ª ed., Mestre Jou: São Paulo, 1981, cap. III.

27. Feigl, F.; Spot Tests In Inorganic Analysis, Elsevier: Amsterdam, 1958, cap. 3.

28. Lurie, J.; Handbook of Analytical Chemistry, Mir Publishers: Moscou, 1978, caps. 3, 6 e 10.

29. Os preços citados são uma média das cotações obtidas a partir de quatro fornecedores.

30. Shriner, R. L.; Fuson, R. C.; Curtin, D. Y.; Morrill, T. C.; The Systematic Identification of Organic Compounds, $6^{\text {th }}$ ed., John Wiley \& Sons: New York, 1980.

31. Afonso, J. C.; Noronha, L. A.; Felipe, R. P.; Freidinger, N.; Quim. Nova 2003, 26, 602 . 